\title{
INTERNETWORKING OF SOLAR ENERGY USING ARDUINO BASED TRACKING SYSTEM
}

\author{
Ms. Aparajita Gogoi \\ MCA scholar,School of CS \& IT, Dept of MCA,Jain (Deemed-to-be) University, Bangalore
}

Article DOI: https://doi.org/10.36713/epra4328

ABSTRACT
Since the dawn of civilization, human beings have been using different energy sources in order to fulfil their
various requirements. Energy is one of the human civilization's basic requirements for development. For example
- electricity powers our household appliances like TV, air-conditioners, phones, washing machines etc. We also
use energy to run vehicles like cars, buses and trains and it is used to run most industries and factories. Most of
these energy sources are non-renewable in nature, and might get depleted soon, such as the Oil and coal. This
creates a requirement for renewable energy resources which has cheap costs. Solar energy is considered as one of the
main energy resources in warm countries. Also solar energy is a clean, easily accessible and abundantly available
alternative energy source in nature and has been used traditionally since ages in the absence of energy sources.
Hence we require a system which will be able to efficiently utilize solar energy such as solar panels but these have
some drawbacks as they don't rotate with the movement of the sun, hence the need arises for that of a solar tracker.
KEYWORDS- IoT, Ldr, Servomotors, Solar Panel

\section{INTRODUCTION}

Solar energy using photovoltaic cells has already been prevalent in our society but they did not take into account the difference of angle of incidence of the sun by providing the solar panels in a fixed orientation. Because in a fixed orientation the amount of energy collected is limited hence the utilization of energy will be limited. The efficiency of the solar 3 . panel can be increased by tracking and following the movement of the sun ie. The photovoltaic cells must be perpendicular to the sun at all the time. The project discusses the development of two-axis solartracking developers using Arduino Uno as main controller the system and four light-dependent resistors (LDRs) have been used for sunlight detection and to get maximum light intensity. Two servo motors have been used to rotate the solar panel according to the sun's light source detected by the LDR and the microcontroller (Arduino Uno R3) is used to control the servo motor based on signals received from the LDRs.

\section{OBJECTIVES}

1. The system which will be able to efficiently utilize solar energy at the maximum level.

2. The proposed system will be able to increase efficiency of the solar panel by tracking and following the movement of the sun.

\section{PROBLEMS WITH EXISTING SYSTEMS}

1. Solar energy using photovoltaic cells have already been prevalent in our society but they did not take into account the difference of angle of incidence of the sun where the panels are fixed. Because in a fixed position the amount of energy collected is limited hence the utilization of energy will be limited.

2. A system is required which will be able to efficiently utilize solar energy. Solar panels as they don't rotate with the movement of the sun, tracking systems must be put in place to move 


\section{EPRA International Journal of Research and Development (IJRD)

the panels throughout the day in order to keep them aligning the sun. The longer they are aligned with the sun, the more energy they can produce.

\section{LITERATURE REVIEW}

A solar cell is a device which converts light energy to electrical energy through photovoltaic effect. Solar cells are the building blocks of photovoltaic modules known as solar panels. In solar tracking system, the module's surface tracks the position of the sun automatically as the day runs by. The position of the sun varies as the sun moves across the sky. For a solar powered equipment to work best it must be placed near the sun and the solar tracker can increase the efficiency of that equipment at any fixed position. However, tracking with great effect can improve both the amount of total output power produced by a system and that produced during critical system demand periods.[1] Researches have been done to improve the energy production of solar panels. These researches include; double-sided panels , conversion stages improvement, building panels integration geometrically and so on. Maximum energy is produced by a solar PV panel when it is positioned at right angle to the sun.[2] For this reason, several researches developed different types of solar panel tracking systems. Therefore, the primary purpose of this work is to develop a solar panel tracker based on Arduino advances so as to enhance the energy production of solar panel.

\section{PROBLEM STATEMENT}

Since solar panels are fixed to a surface, so when the sun moves away from the postion that is facing the solar panel, the power generated is less and looses the opportunity to generate maximum possible power all day long. This project's objective is to have a solar panel which provides its maximum possible power for the whole day, this will only happen when the panel tracks the sun continuously and rotates accordingly, to receive sunlight to the fullest extent always during the day time.

\section{SOLUTION}

Photovoltaic panels or solar panels must be perpendicular with the sun in order to get maximum energy. The methodology employed in this work includes the implementation of an Arduino based solar tracking system. Light Dependent Resistors (LDRs) are used to sense the intensity of sunlight and hence the PV solar panel is adjusted accordingly to track maximum energy. The mechanism uses servo motor to control the movement of the solar panel. The microcontroller is used to control the servo motor based on signals received from the LDRs. The result of this work has shown that the tracking solar panel produces more energy compared to a fixed panel.

\section{IMPLEMENTATION}

\subsection{Working}

The LDRs serve as the sensors to detect the intensity of light entering the solar panels. The LDR then sends information to the Arduino microcontroller. After receiving the information the Arduino microcontroller will tell the servo motor to rotate in a particular direction to face the sun. The solar attached is attached to the servo motor. The microcontroller is attached to a power supply(laptop or battery)

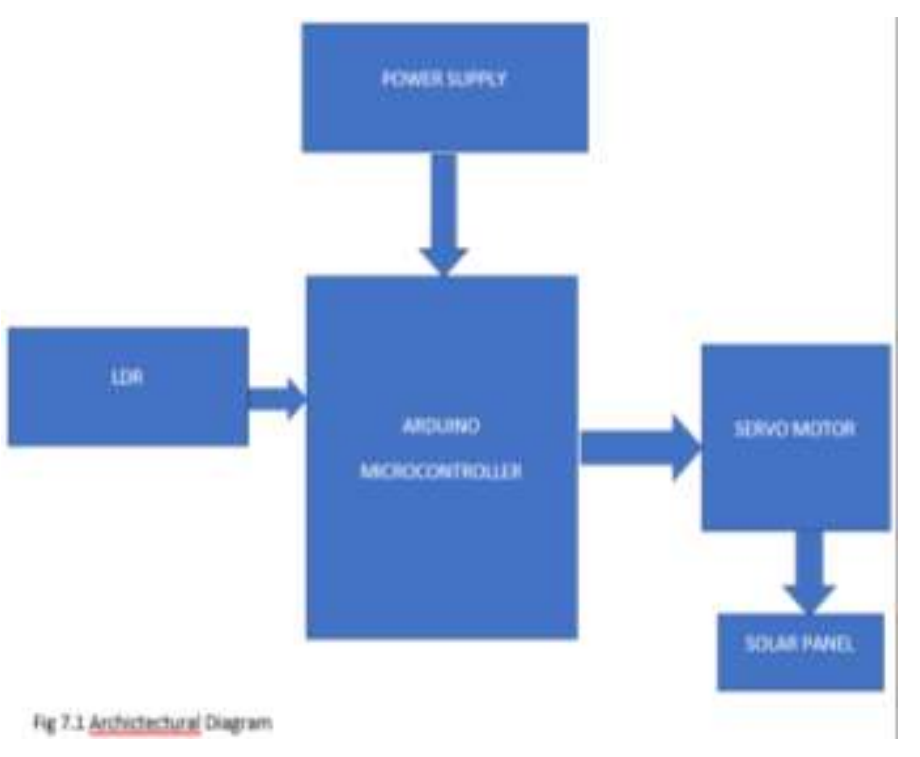




\section{EPRA International Journal of Research and Development (IJRD)}

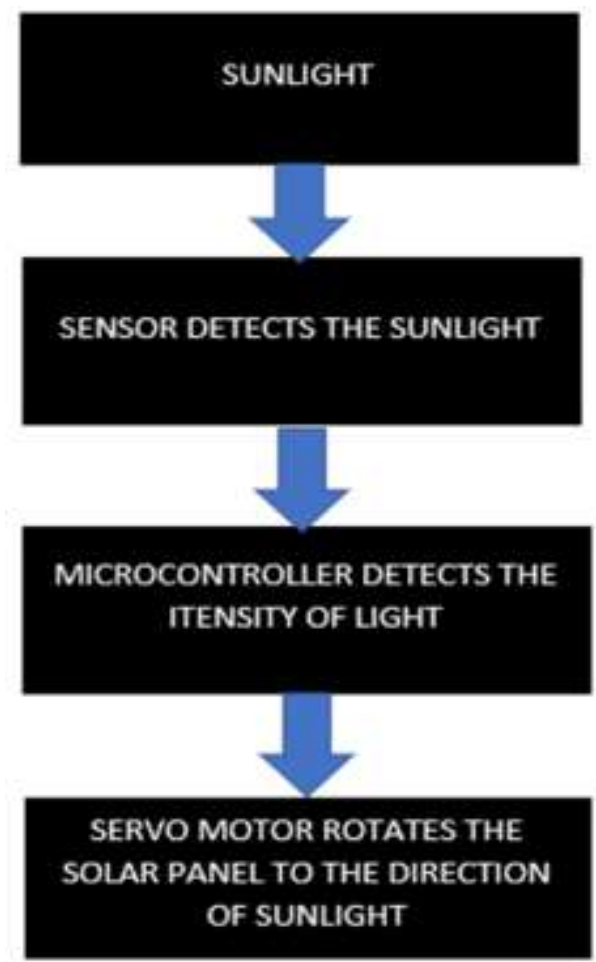

Fig: Flow Diagram

\section{FUTURE ENHANCEMENTS}

A. More sensors and motors can used to give better efficiency and accuracy.

B. A temperature and humidity module(DHT11) can be implemented to get the correct temperature readings. The DHT11 is a commonly used Temperature and humidity sensor. The sensor comes with a dedicated NTC to measure temperature and an 8-bit microcontroller to output the values of temperature and humidity as serial data. The sensor is also factory calibrated and hence easy to interface with other microcontrollers.

C. A RTC modules are simply TIME and DATE remembering systems which have battery setup which in the absence of external power keeps the module running. This will help to keep the TIME and DATE up to date when it is implemented..

D. The ESP8266 WiFi Module is a self contained SOC with integrated TCP/IP protocol stack that can give any microcontroller access to our $\mathrm{WiFi}$ network, this can be implemented for connecting to the internet or cloud in order to store data of the solar panel. This module can be implemented in the future.

E. Changes in the hardware structure can be made such as the structure that holds the panel.

\section{CONCLUSION}

This proposed system is capable to check and pursue the intensity of Sun light and accordingly finds higher energy as result inconsiderate motion of the motor. The aim of this project is fulfilled with present time and reserves. This presented work is also suitable for big size of solar system. In coming days, any investor may apply extra capable sensors and more motors to move the panel according to their discretion. Again this would improve capability of the panel to provide more energy.

\section{REFERENCES}

1. Prachi Rani, Omveer Singh, Shivam Pandey “An Analysis on Arduino based Single Axis Solar Tracker",2018

2. Peter Amaize, Stanley Uzairue, Timilehin Fiyin Sanni “ Arduino Based Solar Tracking System For Energy Improvement of Pv Solar Panel",2018

3. Mohamad Nur Aiman Mohd Said, Siti Amely Jumaat, Clarence Rimong Anak Jawa, "Dual axis solar tracker with IoT monitoring system using Arduino",2019

4. Divya Gnanarathinam., Sundaramurthy, Amitabh Wahi, "Design and Implementation of a Dual Axis Solar Tracking System", 2018 